\title{
A Simple Technique for Improving the IM3/C and PAE Performance of MESFET Amplifiers
}

\author{
J N H Wong and C S Aitchison \\ Microwave Systems Research Group, Advanced Technology Institute, University of Surrey, \\ Guildford, Surrey GU2 7XH, UK
}

\begin{abstract}
This paper shows by simulation that a shunt short -circuited $\lambda / 4$ line placed across the drain terminals of a microwave MESFET amplifier significantly improves both the IM3/C and 2-tone PAE performance by a maximum of $14 \mathrm{~dB}$ and $3 \%$ (from $24.5 \%$ to $27.5 \%$ ), respectively. Practical confirmation with both WCDMA and GSM-EDGE input signals is obtained with a microstrip amplifier at $2 \mathrm{GHz}$ demonstrating an average improvement in ACPR of 12.5dB and a reduction in EVM from $5.0 \%$ to $1.3 \%$ respectively. The technique is novel, simple and practical and will be of direct interest to designers of base station amplifiers.
\end{abstract}

\section{INTRODUCTION}

There has recently been interest in improving the linearity of microwave MESFET power amplifiers for cellular telephone communication systems and their requirements for good linearity and efficiency.

Historically a large number of techniques have been devised to improve amplifier intermodulation behaviour [1]. These techniques are all complicated in comparison with the novel technique described in this paper.

There are three significant non-linearities associated with the behaviour of a MESFET amplifier: they are the $\mathrm{g}_{\mathrm{m}}$ and $\mathrm{C}_{\mathrm{gs}}$ associated with the gate and $\mathrm{g}_{\mathrm{d}}$ associated with the drain. Previous theoretical and experimental work have demonstrated that injection of the second harmonic of the signal input components into the gate, with the correct phase and amplitude can improve the 2-tone intermodulation performance [2], [3]. Alternatively injection of the frequency separation between the tones of the 2-tone input signal (here referred to as the difference frequency) also improves the 2-tone intermodulation performance [3], [4], [5].

This experience suggests that provision of an appropriate short circuit impedance across the drain terminal of the active device at a relevant frequency such as the difference frequency or the second harmonic frequencies might improve the intermodulation and PAE performance significantly.

This paper describes an investigation of this proposition first by simulation and secondly by construction and measurement of a $2 \mathrm{GHz}$ MESFET amplifier.

\section{SIMULATION}

Agilent ADS was used to simulate the non linear behaviour and 2-tone PAE of a microstrip amplifier operating at $2 \mathrm{GHz}$ using a Mitsubishi MGF2430A 1 watt GaAs MESFET biased at Class AB/B using a large signal model from the ADS non-linear MESFET library.

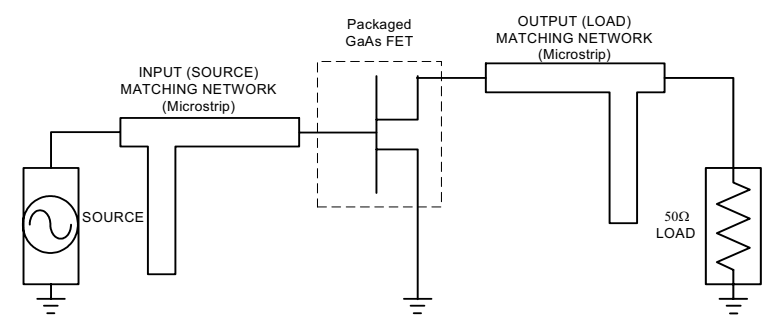

Fig. 1. 2GHz amplifier without special drain termination

Fig. 1 shows the basic amplifier without the addition of special drain termination components and it can be seen that matching is provided at both the gate and the drain by means of microstrip lines and open-circuit stubs. This basic amplifier is described as the reference amplifier. The simulated small signal gain of the reference amplifier was $14 \mathrm{~dB}$ at a centre frequency of $2 \mathrm{GHz}$ with input and output return losses of $-18.3 \mathrm{~dB}$ and $-7.7 \mathrm{~dB}$ respectively. The amplifier was simulated at a drain bias of 6 volts and a gate bias of -2.1 volts.

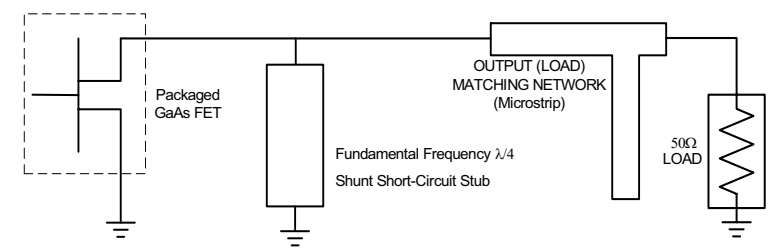

Fig. 2. 2GHz amplifier output circuit showing shorted shunt $\lambda / 4$ line at input frequency placed at the drain

Fig. 2 shows a modified version of the amplifier output configuration in which a $\lambda / 4$ shunt short circuited stub at 
the input frequency is placed at the drain terminal. This will not affect the impedance at the input frequency but will provide a short circuit at the second harmonic and some other frequencies.

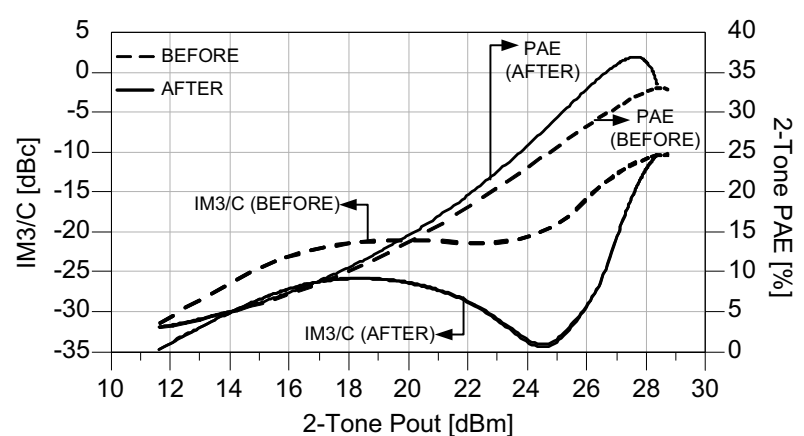

Fig. 3. Simulated IM3/C (both upper and lower) and 2-tone PAE as function of output power for both the $2 \mathrm{GHz}$ amplifier with shorted $\lambda / 4$ drain stub and for the reference amplifier.

Fig. 3 shows the predicted 2-tone IM3/C and 2-tone PAE for this amplifier configuration as a function of 2tone output power dissipated in the $50 \Omega$ load, together with the corresponding plots for the reference amplifier. These results show a $14 \mathrm{~dB}$ maximum improvement in IM3/C and a corresponding 2-tone PAE improvement of $3 \%$ (from $24.5 \%$ to $27.5 \%$ ). This result is of interest but requires further investigation since the $\lambda / 4$ stub presents non-infinite shunt impedances at frequencies which may be relevant to the amplifier non-linear behaviour.

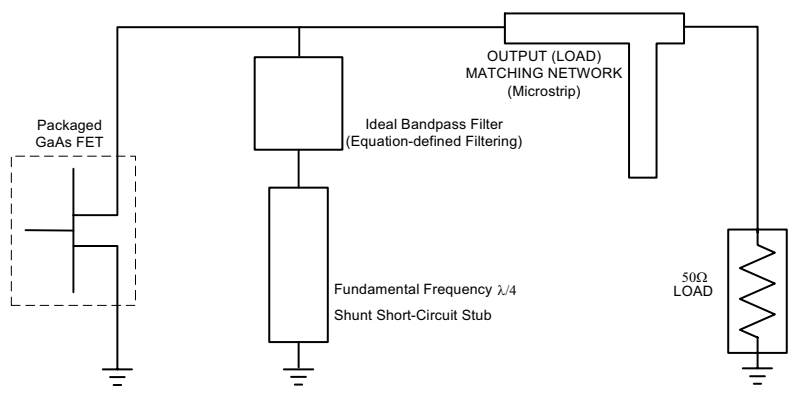

Fig. 4. Output circuit of $2 \mathrm{GHz}$ amplifier with equation defined BPF preceding shorted shunt input frequency $\lambda / 4$ stub

A convenient way of determining the significance of the various relevant frequencies is shown in Fig. 4. An equation-defined ideal BPF precedes the $\lambda / 4$ fundamental frequency short-circuited stub. Variation of the filter centre frequency and bandwidth enables the effect of the impedances at various frequencies presented by the $\lambda / 4$ stub to be investigated. The frequencies selected for investigation were the two $2^{\text {nd }}$ harmonics, difference, and combined second harmonics and difference frequencies

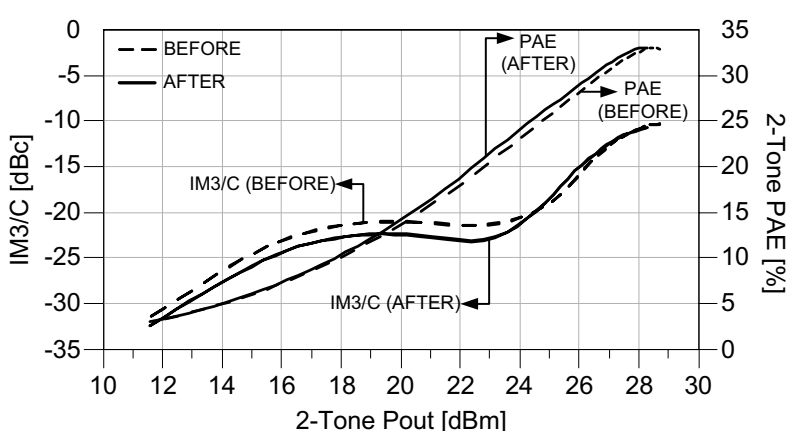

Fig.5. Simulated IM3/C (both upper and lower) and 2-tone $\mathrm{PAE}$ as function of output power for $2 \mathrm{GHz}$ amplifier with $2^{\text {nd }}$ harmonic BPF preceding the shorted shunt input frequency $\lambda / 4$ line compared with the reference amplifier

Fig 5 shows the behaviour of the amplifier with the BPF at the second harmonic frequencies and shows a maximum improvement of only $2 \mathrm{~dB}$ in IM3/C and $1.0 \%$ (from $19.5 \%$ to $20.5 \%$ ) in 2-tone PAE at an output of $22.5 \mathrm{dBm}$.

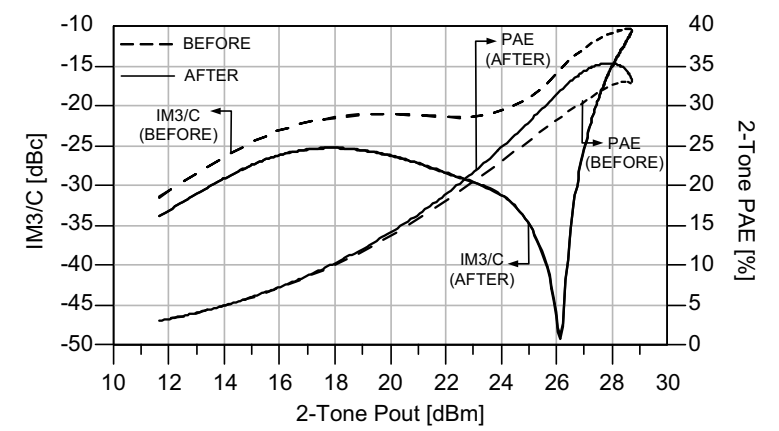

Fig. 6. Simulated IM3/C and 2-tone PAE as function of output power for $2 \mathrm{GHz}$ amplifier with difference frequency BPF preceding shorted $\lambda / 4$ drain stub compared with reference amplifier.

Fig. 6 shows the corresponding behaviour of the amplifier with the BPF centred on the difference frequency. This configuration gives a maximum improvement in IM3/C of $33 \mathrm{~dB}$ and an improvement in 2tone PAE of $4 \%$ (from $28 \%$ to $32 \%$ ) at an output power of $26 \mathrm{dBm}$.

Fig. 7 shows the behaviour of the amplifier with an equation defined BPF configuration centred on both the difference and second harmonic frequencies. The maximum IM3/C improvement is now $14 \mathrm{~dB}$ : the 2-tone PAE has improved by $3.0 \%$ (from $23.5 \%$ to $26.5 \%$ ) Both results are in substantial agreement with the $\lambda / 4$ stub result of Fig. 3. 


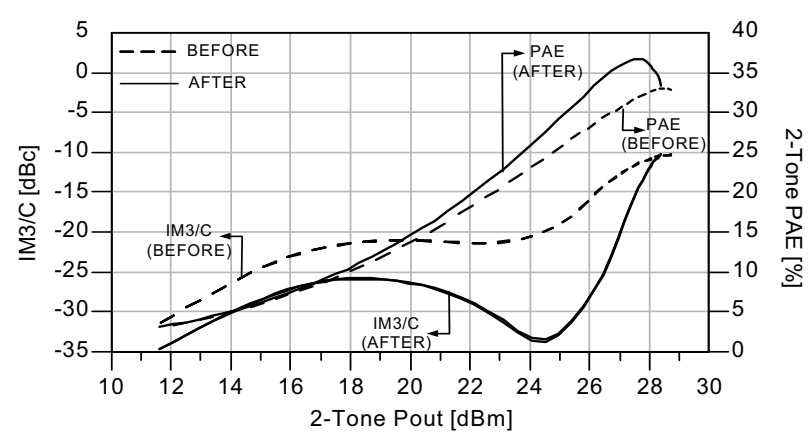

Fig. 7. Simulated IM3/C (both) and 2-tone PAE as function of output power for with BPF centered on the difference and $2^{\text {nd }}$ harmonic frequencies, and reference amplifier.

These simulations suggest that the $\lambda / 4$ stub improvement in $\mathrm{IMC} / 3$ and 2-tone PAE is due primarily to the provision of an effective short circuit at the difference frequency. It also seems that the presence of the shunt short circuit at the difference frequency and second harmonics together result in a useful but reduced improvement in performance.

The next section describes a practical investigation.

\section{PRACTICAL INVESTIGATION}

It is convenient to provide a shunt $\lambda / 4$ short circuited stub at the drain of a microstrip amplifier in practice so this configuration was selected for practical investigation at $2 \mathrm{GHz}$ as shown in Fig. 8. A Mitsubishi MGF 2430A 1 watt GaAs MESFET with 6 volt drain and -2.1 volt gate bias was used

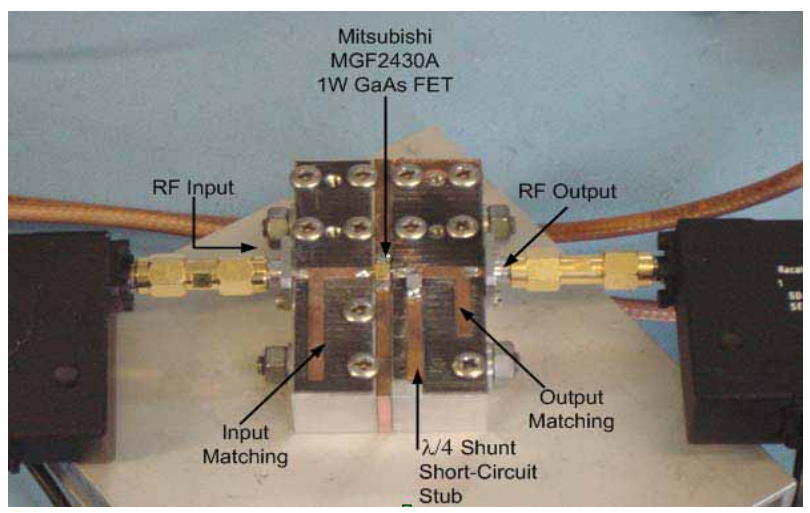

Fig. 8. Photograph of $2 \mathrm{GHz}$ microstrio amplifier showing $\lambda / 4$ short-circuited stub at the drain.

A second amplifier without the drain stub was used as the reference amplifier and the MESFET was moved between the two amplifiers for performance comparison..

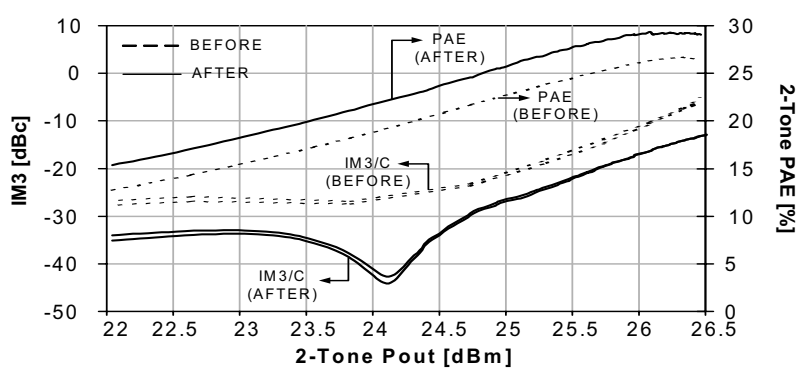

Fig. 9. Measured IM3/C and PAE for $2 \mathrm{GHz}$ amplifier with shorted $\lambda / 4$ drain stub and reference amplifier as function of output power. Both upper and lower IM3/C plots are shown.

Fig 9 shows the measured non linear performance and PAE of the practical amplifier with and without the $\lambda / 4$ stub with an input consisting of two tones at $2 \mathrm{GHz}$ separated by $1 \mathrm{MHz}$ and shows results for both upper and lower IM3 products. A maximum improvement in IM3/C of $17 \mathrm{~dB}$ and in 2 -tone PAE of $4 \%$ (from $18 \%$ to $22 \%$ ) was measured at an output power of $24.2 \mathrm{dBm}$.

The performance of the amplifier with WCDMA and GSM-EDGE input signals was then examined.

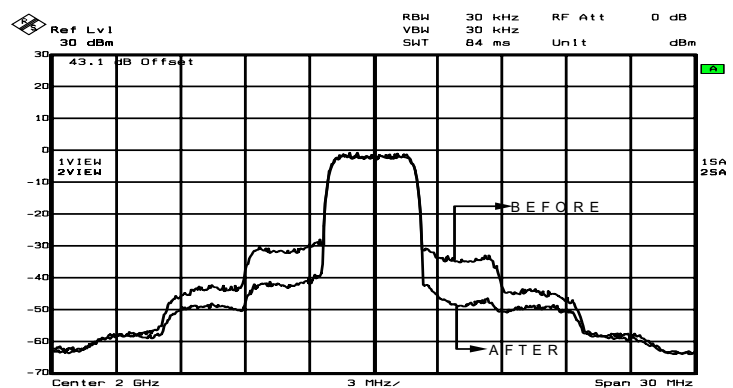

Fig. 10. Measured Spectrum of $2 \mathrm{GHz}$ amplifier with shorted $\lambda / 4$ drain stub and reference amplifier with WCDMA input.

Fig. 10 shows the spectrum of the amplifier output with a WCDMA input illustrating an average ACPR improvement of $12.5 \mathrm{~dB}$ at an offset of $5.0 \mathrm{MHz}$ for an average output power of $19.5 \mathrm{dBm}$.

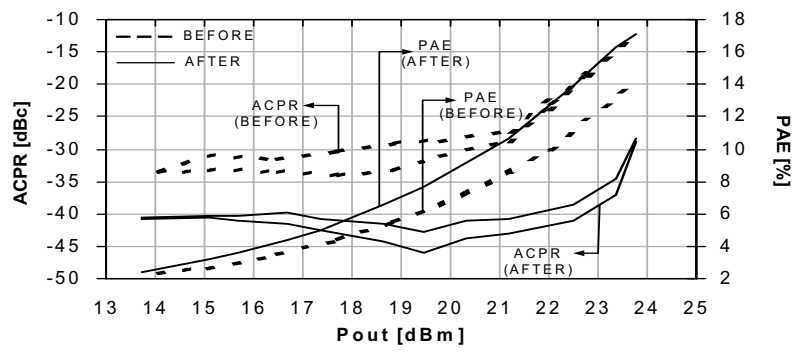

Fig. 11. Variation of ACPR and PAE with output power for amplifier with shorted $\lambda / 4$ drain stub and reference amplifier both with WCDMA input. 
The variation of ACPR and PAE with average power output is shown in Fig 11 for a WCDMA input and shows a maximum improvement in average ACPR of about $13 \mathrm{~dB}$ at an average power output of $19.5 \mathrm{dBm}$. This improvement increases for higher average power output. The PAE increases by $1.5 \%$ (from $6.2 \%$ to $7.7 \%$ ) at the same output power.

The corresponding amplifier spectrum performance with a GSM-EDGE signal input is shown in Fig. 12 for an average power output of $20 \mathrm{dBm}$ and shows an improvement of about $9 \mathrm{~dB}$ at $250 \mathrm{kHz}$ offset and $7 \mathrm{~dB}$ at $400 \mathrm{kHz}$ offset.

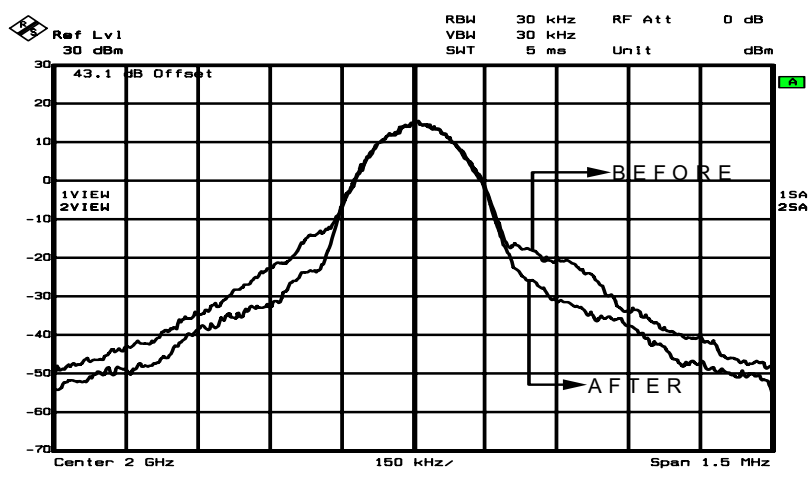

Fig. 12. Measured Spectrum of $2 \mathrm{GHz}$ amplifier with shorted $\lambda / 4$ drain stub and reference amplifier with GSM- EDGE input.

The variation of the GSM-EDGE ACPR at an offset of $250 \mathrm{kHz}$ as a function of average power output for both upper and lower channels is given in Fig. 13 and shows an improvement of about $8 \mathrm{~dB}$ at an output power of $20 \mathrm{dBm}$. The $400 \mathrm{kHz}$ offset results show a reduced improvement of $6.5 \mathrm{~dB}$.

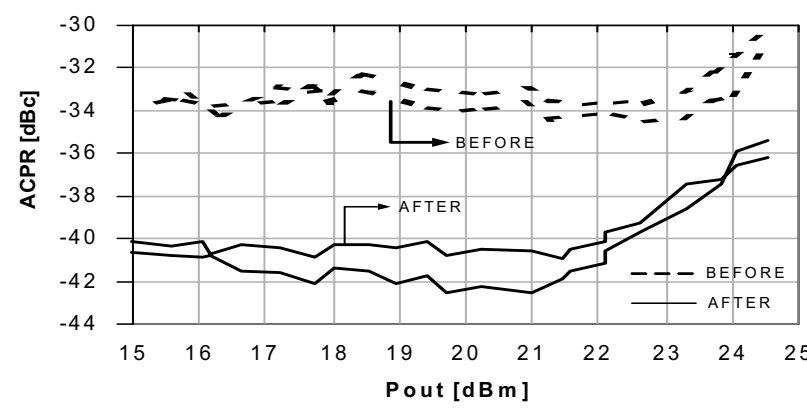

Fig. 13. Variation of ACPR with GSM-EDGE input for amplifier with shorted $\lambda / 4$ drain stub, and reference amplifier

Fig. 14 illustrates the measured EVM and PAE as a function of average power output showing that at $20 \mathrm{dBm}$ output the EVM decreases from $5.0 \%$ to $1.3 \%$ and the PAE improves by $1.0 \%$ (from $7.5 \%$ to $8.5 \%$ ).

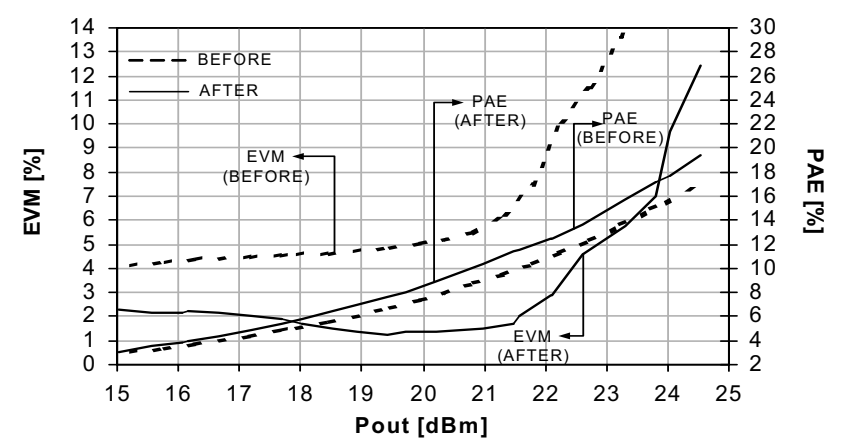

Fig. 14. Variation of EVM with GSM-EDGE input for amplifier with shorted $\lambda / 4$ drain stub, and reference amplifier, as function of output power.

\section{CONCLUSION}

Simulation shows that the predicted $2 \mathrm{GHz}$ Class $\mathrm{A} / \mathrm{AB}$ amplifier performance improvement, compared with a reference amplifier, in 2-tone IM3/C and PAE due to a drain $\lambda / 4$ shorted-stub is $14 \mathrm{~dB}$ and $3 \%$ (from $24.5 \%$ to $27.5 \%$ ) respectively. A short circuit at the difference frequency improves these figures to $33 \mathrm{~dB}$ and $4 \%$ (from $28 \%$ to $32 \%$ ). These predictions are for output powers about $3 \mathrm{~dB}$ below saturation

Practical measurement on a $2 \mathrm{GHz}$ microstrip amplifier with a $\lambda / 4$ shorted stub show an improvement of $17 \mathrm{~dB}$ in $\mathrm{IM} 3 / \mathrm{C}$ and $4 \%$ (from $18 \%$ to $22 \%$ ) in 2-tone PAE compared with the reference practical amplifier. The APCR with a WCDMA signal is improved by $12.5 \mathrm{~dB}$ at an off-set of $5.0 \mathrm{MHz}$ for an output power of $19.5 \mathrm{dBm}$. With a GSM-EDGE signal the ACPR improves by $8 \mathrm{~dB}$ for an off-set of $250 \mathrm{kHz}$ The corresponding EVM improves from $5.0 \%$ to $1.3 \%$.

\section{REFERENCES}

[1] C. S. Aitchison, "The current status of RF and microwave amplifier intermodulation performance," IEEE Radio Frequency Int. Crts. Sym., Boston, June 2000, pp.113-116.

[2] F.Ali,M.R.Moazzam, and C.S.Aitchison, "IMD elimination and APCR improvement for a $800 \mathrm{MHz}$ HBT MMIC power amplifier,"IEEE RFIC Sym. Digest, vol 2, Baltimore, MD, June 1998,pp. 69-71

[3] C.S.Aitchison et al, " Improvement of $3^{\text {rd }}$ order Intermodulation product of RF and microwave amplifiers by injection", IEEE. Trans. MTT, June 01, vol.49, pp.11481154 .

[4] M.Modeste et al, " Analysis and practical performance of a difference frequency technique for improving the multicarrier IMD performance of RF amplifiers," MTT. Wireless App. Sym. Dig, Vancouver, ,Feb.99, pp53-56.

[5] W.J.Jenkins and A.Khanifer, " A multicarrier amplifier with low third-order intermodulation distortion," IEEE MTT-S Dig., vol. 3, Boston, June 2000, pp. 1515-1518. 